\title{
Effects of a near-field rigid sphere scatterer on the performance of linear microphone array beamformers
}

\author{
Yuxiang $\mathrm{Hu},{ }^{\text {a) }}$ Haoran Zhou, Jing $\mathrm{Lu}^{\text {, b) }}$ and Xiaojun $\mathrm{Qiu}^{\mathrm{a})}$ \\ Key Laboratory of Modern Acoustics, Institute of Acoustics, Nanjing University, Nanjing 210093, China
}

(Received 5 February 2016; revised 13 July 2016; accepted 13 July 2016; published online 11 August 2016)

Beamformers enable a microphone array to capture acoustic signals from a sound source with high signal to noise ratio in a noisy environment, and the linear microphone array is of particular importance, in practice, due to its simplicity and easy implementation. A linear microphone array sometimes is used near some scattering objects, which affect its beamforming performance. This paper develops a numerical model with a linear microphone array near a rigid sphere for both far-field plane wave and near-field sources. The effects of the scatterer on two typical beamformers, i.e., the delay-and-sum beamformer and the superdirective beamformer, are investigated by both simulations and experiments. It is found that the directivity factor of both beamformers improves due to the increased equivalent array aperture when the size of the array is no larger than that of the scatter. With the increase of the array size, the directivity factor tends to deteriorate at high frequencies because of the rising side-lobes. When the array size is significantly larger than that of the scatterer, the scattering has hardly any influence on the beamforming performance.

(C) 2016 Acoustical Society of America. [http://dx.doi.org/10.1121/1.4960546]

[MRB]

Pages: 924-935

\section{INTRODUCTION}

Microphone arrays have been widely studied in speech communication applications, such as hands-free telephony, hearing aids, speech recognition, and enhancement. ${ }^{1-3} \mathrm{~A}$ basic function of microphone arrays is the beamformer, which is designed to capture desired signals from the socalled look direction and suppress the interference from all the other directions. Various array configurations have been used for the beamformers design. ${ }^{4-6}$ Among them, linear microphone arrays are widely used and studied due to its simplicity and easy implementation.

Conventional approaches for designing beamformers usually assume ideal or known microphone characteristics. ${ }^{4,7}$ However, these beamformers are sensitive to the errors in microphone characteristics. ${ }^{8}$ To combat this problem, several robust beamformer design approaches have been proposed in recent years. ${ }^{8-14}$ In Refs. $8-10$, robustness is improved by optimizing the mean performance, where the probability density function of microphone characteristics is taken into consideration. Another popular approach is based on the minimax design criterion with the worst-case performance optimization. ${ }^{11-13}$ The white noise gain constraint approach is also widely used in robust superdirective beamformers design..$^{9,14}$

Most commonly used robust design approaches only consider the random errors in the microphone array characteristics. However, the non-ideal acoustic field inevitably influences the beamformer performance, which is of significance to be investigated. In this paper, deviations caused by

\footnotetext{
a) Also at: School of Electrical and Computer Engineering, RMIT University, Melbourne 3000, Australia.

b)Electronic mail: lujing@nju.edu.cn
}

the scattering from a near-field scatterer are of particular interest. Acoustic scattering of a near-field human head have been considered in head mounted arrays, such as hearing aids, headsets and helmets. ${ }^{15-17}$ Using a uniform circular array mounted on a rigid sphere, the scattering effect by the rigid sphere can be utilized to design beamformers with narrower beamwidth and higher directivity factor (DF). ${ }^{18,19}$ However, the influence of the near-field scatterer on the performance of the linear microphone array beamformers has yet to be analyzed.

In this paper, a near-field scattering model is established, where the near-field scatterer is assumed as a rigid sphere. Both the far-field plane wave model and the near-field point source model are used in the analysis. Furthermore, a reasonable analytical model considering the scattering of the speaker is utilized. The time domain simulation is used to analyze the influence of the scattering on the wave propagation, and then the influence of the near-field scatterer on the performance of two typical beamformers, i.e., the delay-and-sum (DS) and the superdirective beamformers, ${ }^{1}$ are investigated. The DF of arrays under four different configurations with and without the scattering are compared, and some meaningful features together with the physical explanations are presented. The experiments using arrays and rigid spheres under these different configurations further validate the features.

\section{THEORETICAL MODELS}

Figure 1 shows the geometrical arrangements of the beamforming system with a scatterer, where the scatterer is assumed to be a rigid sphere. The region of interest is the horizontal plane including the center of the rigid sphere and the line of the linear microphone array. In the following derivations, Cartesian coordinate system $(x, y, z)$ and spherical 
coordinate system $(r, \theta, \varphi)$ are considered, where $r, \theta$, and $\varphi$ represent radius, elevation, and azimuth, respectively.

\section{A. Far-field sound source model with a scatterer}

When the sound source is far from the array, the incident wave is usually assumed as plane waves as depicted in Figs. 1(a) and 1(b). In spherical coordinates, the incident plane wave can be expressed as ${ }^{21}$

$$
\begin{aligned}
p_{i}(r, \theta) & =p_{0} e^{-\mathrm{j} k r \cos \theta} \\
& =p_{0} \sum_{l=0}^{\infty}(-\mathrm{j})^{l}(2 l+1) j_{l}(k r) P_{l}(\cos \theta),
\end{aligned}
$$

where $\mathrm{j}=(-1)^{1 / 2}, p_{0}$ is the amplitude of the incident wave, $k$ is the wave number, $j_{l}(\cdot)$ is the spherical Bessel function of order $l$, and $P_{l}(\cdot)$ is the Legendre function of order $l$.

The total sound field with consideration of the rigid sphere scattering can be expressed as ${ }^{21,22}$

$$
\begin{aligned}
p_{\text {tot }}(r, \theta)= & p_{0} \sum_{l=0}^{\infty}(-\mathrm{j})^{l}(2 l+1) \\
& \times\left[j_{l}(k r)-\frac{j^{\prime}{ }_{l}(k a)}{h^{\prime}{ }_{l}(k a)} h_{l}(k r)\right] P_{l}(\cos \theta),
\end{aligned}
$$

where $a$ is the radius of the rigid sphere, $h_{l}(\cdot)$ is the spherical Hankel function of order $l, j_{l}^{\prime}(\cdot)$, and $h_{l}^{\prime}(\cdot)$ are the derivatives of the spherical Bessel and Hankel function, respectively.
For the far-field plane wave, the sound pressure at each microphone can be calculated by Eq. (2), then the performance of different beamforming algorithms with plane wave assumption under the influence of the scattering can be simulated.

\section{B. Point source model with a scatterer}

When the sound source is located at the near-field region of the array, the plane wave assumption is no longer valid and the point source model is used. ${ }^{4}$ In spherical coordinates, the sound pressure at location $\mathbf{r}_{e}=\left(r_{e}, \theta_{e}, \varphi_{e}\right)$ generated by a point source located at $\mathbf{r}_{c}=\left(r_{c}, \theta_{c}, \varphi_{c}\right)$ can be expanded in terms of spherical harmonics ${ }^{21}$

$$
\begin{aligned}
p_{i}\left(\boldsymbol{r}_{e}\right)= & \mathrm{j} \omega \rho_{0} q \frac{e^{-\mathrm{j} k r}}{4 \pi r} \\
= & k \omega \rho_{0} q \sum_{l=0}^{\infty} j_{l}\left(k r_{<}\right) h_{l}\left(k r_{>}\right) \\
& \times \sum_{m=-l}^{l} Y_{l m}^{*}\left(\theta_{c}, \varphi_{c}\right) Y_{l m}\left(\theta_{e}, \varphi_{e}\right),
\end{aligned}
$$

where $\omega$ is the angular frequency, $\rho_{0}$ is the mean air density, $q$ is the volume velocity of the source, $r=\left|\mathbf{r}_{e}-\mathbf{r}_{c}\right|, Y_{l m}$ is the spherical harmonic of order $l$ and degree $m, \mathbf{r}_{<}=\min \left(\left|\mathbf{r}_{e}\right|\right.$, $\left.\left|\mathbf{r}_{c}\right|\right)$ and $\mathbf{r}_{>}=\max \left(\left|\mathbf{r}_{e}\right|,\left|\mathbf{r}_{c}\right|\right)$. The superscript (*) denotes complex conjugation.

The total sound field with consideration of the rigid sphere scattering can be expressed as ${ }^{21,22}$

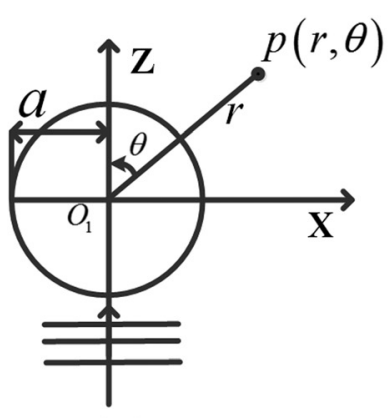

(a)

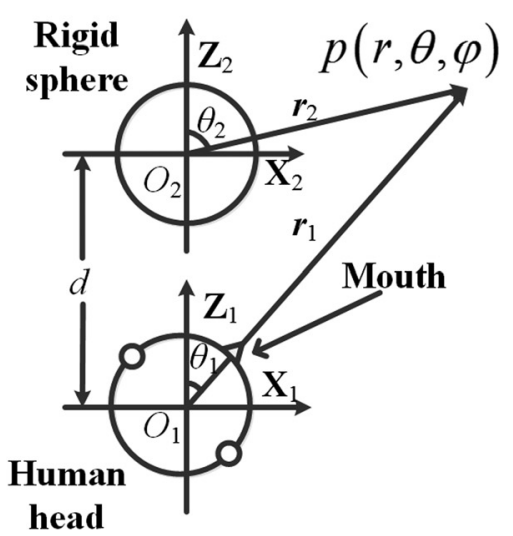

(c)

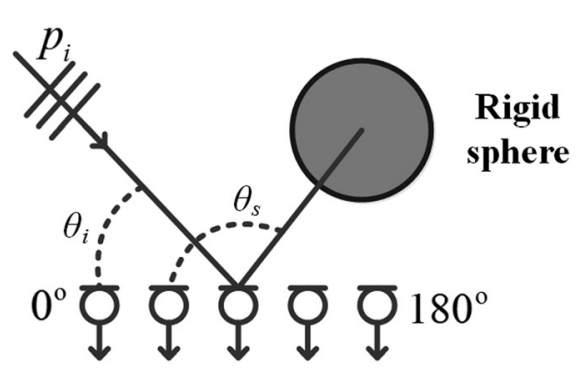

(b)

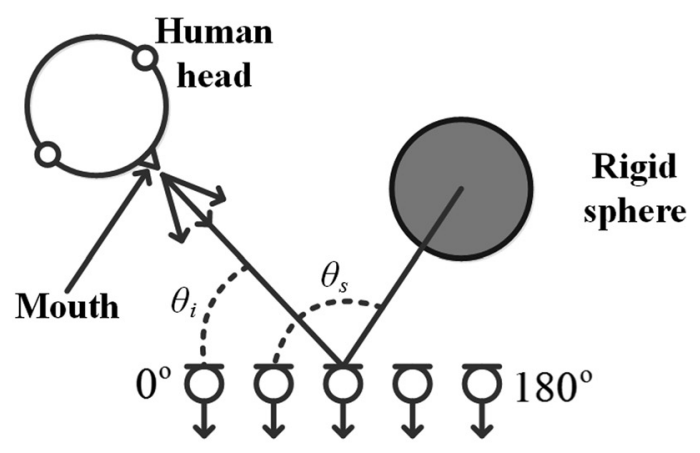

FIG. 1. (a) Geometry of the plane wave and a rigid sphere, (b) geometry of the plane wave, the rigid sphere, and the microphone array, (c) geometry of a speaker and a rigid sphere, (d) geometry of the speaker, the rigid sphere, and the microphone array. 


$$
\begin{aligned}
p_{\text {tot }}\left(\mathbf{r}_{e}\right)= & k \omega \rho_{0} q \\
& \times \sum_{l=0}^{\infty}\left[j_{l}\left(k r_{<}\right) h_{l}\left(k r_{>}\right)-\frac{j^{\prime}{ }_{l}(k a)}{h^{\prime}(k a)} h_{l}\left(k r_{c}\right) h_{l}\left(k r_{e}\right)\right] \\
& \times \sum_{m=-l}^{l} Y_{l m}^{*}\left(\theta_{c}, \varphi_{c}\right) Y_{l m}\left(\theta_{e}, \varphi_{e}\right) .
\end{aligned}
$$

For the near-field point source, the sound pressure at each microphone can be calculated by Eq. (4), then the performance of different beamforming algorithms with point source assumption under the influence of the scattering can be simulated.

\section{A speaker model with a scatterer}

Considering that the microphone array is usually used to capture the voice of a speaker, a proper speaker model instead of the point source will lead to a more reasonable near-field model. In this paper, an analytical speaker model, in which the speaker's head is represented by a rigid sphere and the speaker's mouth is represented by a radially vibrating piston mounted on the surface of the rigid sphere, ${ }^{21}$ is utilized. The basic schematic diagram of the speaker model together with the rigid sphere scatterer is shown in Fig. 1(c). Two spherical coordinate systems are used in this diagram, referred to as $O_{1}$ and $O_{2}$, respectively. A receiver point is located at $\mathbf{r}_{1}=\left(r_{1}, \theta_{1}, \varphi_{1}\right)$ in the coordinate system $O_{1}$ or at $\mathbf{r}_{2}=\left(r_{2}, \theta_{2}, \varphi_{2}\right)$ in the coordinate system $O_{2}$. The $X Z$ planes of both coordinate systems are located within the same plane and in the same axial directions, and therefore $\varphi_{1}=\varphi_{2}$.

The speaker source is modeled as a rigid sphere of radius $b$, and the mouth is modeled as a piston of radius $b \sin \theta_{0}$ located on the surface of a rigid sphere. To simplify the simulations, the mouth of the human head is assumed to face the center of the microphone array, as depicted in Fig. 1(d). The amplitude of the surface velocity of the human head sphere is assumed as

$$
U(\theta, \varphi)=\left\{\begin{array}{cc}
u_{0}, & 0 \leq \theta \leq \theta_{0}, 0 \leq \varphi<2 \pi \\
0, & \theta_{0} \leq \theta \leq \pi, 0 \leq \varphi<2 \pi .
\end{array}\right.
$$

The velocity distribution can be expanded in terms of spherical harmonics as ${ }^{23}$

$$
U(\theta, \varphi)=\sum_{l=0}^{\infty} \sum_{m=-l}^{l} U_{l m} Y_{l m}(\theta, \varphi) .
$$

The spherical harmonic coefficients are given by

$$
U_{l m}= \begin{cases}\frac{u_{0}}{2} \sqrt{\frac{4 \pi}{2 l+1}}\left[P_{l-1}^{0}\left(\cos \theta_{0}\right)-P_{l+1}^{0}\left(\cos \theta_{0}\right)\right], & m=0 \\ 0, & m \neq 0\end{cases}
$$

where $P_{l}^{m}(\cdot)$ is the associated Legendre function of degree $l$ and order $m$, and for $l=0, P_{-1}^{0}(x)=1$. The pure-tone pressure at $\mathbf{r}_{e}$ outside the rigid sphere source can be expressed as $^{21}$

$$
p_{i}\left(\mathbf{r}_{e}\right)=\sum_{l=0}^{\infty}-\mathrm{j} \frac{\rho_{0} c_{0} U_{l 0}}{h_{l}^{\prime}(k b)} h_{l}\left(k r_{e}\right) Y_{l 0}\left(\theta_{e}, \varphi_{e}\right)
$$

After rotating the center of the vibrating piston to a new position $\left(\theta_{D}, \varphi_{D}\right)$, the new spherical harmonic coefficients can be written as ${ }^{23}$

$$
U_{l m}^{D}=\sqrt{\frac{4 \pi}{2 l+1}} U_{l 0} Y_{l m}^{*}\left(\theta_{D}, \varphi_{D}\right) .
$$

Using the identity $P_{l}^{-m}(\cos \theta)=(-1)^{m}[(l-m) ! /(l+m) !]$ $\times P_{l}^{m}(\cos \theta)$, the new velocity distribution can be written as

$$
\begin{aligned}
U^{D}(\theta, \varphi)= & \sum_{l=0}^{\infty} \sum_{m=-l}^{l} U_{l m}^{D} Y_{l m}(\theta, \varphi) \\
= & \sum_{l=0}^{\infty} \sum_{m=0}^{l}\left(2-\delta_{0 m}\right) U_{l 0} \sqrt{\frac{2 l+1}{4 \pi}} \frac{(l-m) !}{(l+m) !} \\
& \times P_{l}^{m}(\cos \theta) P_{l}^{m}\left(\cos \theta_{D}\right) \cos \left[m\left(\varphi-\varphi_{D}\right)\right],
\end{aligned}
$$

where $\delta_{n m}$ represents the Kronecker delta.

The total sound field $p_{\text {tot }}(\mathbf{r})$ consists of two parts: the radiated and scattered sound field from the speaker (Sphere 1) and the scattered field from the scatterer (Sphere 2). It can be expressed as

$$
p_{\text {tot }}(\mathbf{r})=p_{s_{1}}\left(\mathbf{r}_{1}\right)+p_{s_{2}}\left(\mathbf{r}_{2}\right) .
$$

The sound field (radiated and/or scattered) from each sphere can be conveniently expressed in its own coordinate system as

$$
\begin{aligned}
& p_{s_{1}}\left(\mathbf{r}_{1}\right)=\sum_{l=0}^{\infty} \sum_{m=-l}^{l} C^{\prime}{ }_{l m} h_{l}\left(k r_{1}\right) Y_{l m}\left(\theta_{1}, \varphi_{1}\right), \\
& p_{s_{2}}\left(\mathbf{r}_{2}\right)=\sum_{l=0}^{\infty} \sum_{m=-l}^{l} D^{\prime}{ }_{l m} h_{l}\left(k r_{2}\right) Y_{l m}\left(\theta_{2}, \varphi_{2}\right),
\end{aligned}
$$

where $C^{\prime}{ }_{l m}$ and $D^{\prime}{ }_{l m}$ are unknown spherical harmonic coefficients to be determined by applying the boundary conditions on the surfaces of the spheres. Using $C^{\prime}{ }_{l m}=C_{l m} Y_{l m}^{*}\left(\theta_{D}, \varphi_{D}\right)$ and $D_{l m}^{\prime}=D_{l m} Y_{l m}^{*}\left(\theta_{D}, \varphi_{D}\right)$, Eq. (12) can be rewritten as

$$
\begin{aligned}
p_{s_{1}}\left(\mathbf{r}_{1}\right)= & \sum_{l=0}^{\infty} \sum_{m=0}^{l} C_{l m} h_{l}\left(k r_{1}\right) P_{l}^{m}\left(\cos \theta_{1}\right) P_{l}^{m}\left(\cos \theta_{D}\right) \\
& \times \cos \left[m\left(\varphi_{1}-\varphi_{D}\right)\right], \\
p_{s_{2}}\left(\mathbf{r}_{2}\right)= & \sum_{l=0}^{\infty} \sum_{m=0}^{l} D_{l m} h_{l}\left(k r_{2}\right) P_{l}^{m}\left(\cos \theta_{2}\right) P_{l}^{m}\left(\cos \theta_{D}\right) \\
& \times \cos \left[m\left(\varphi_{2}-\varphi_{D}\right)\right] .
\end{aligned}
$$

Equation (11) has to be written in the same coordinate system. This can be achieved by using the translational addition theorem as ${ }^{23-25}$

$$
\begin{gathered}
h_{l}\left(k r_{j}\right) P_{l}^{m}\left(\cos \theta_{j}\right)=\sum_{n=m}^{\infty} A_{m n}^{m l}\left(\mathbf{r}_{j i}\right) j_{n}\left(k r_{i}\right) P_{n}^{m}\left(\cos \theta_{i}\right), \\
i, j=1,2,
\end{gathered}
$$


where $\mathbf{r}_{j i}=\mathbf{r}_{i}-\mathbf{r}_{j}$ and

$$
\begin{aligned}
A_{m n}^{m l}\left(\mathbf{r}_{j i}\right)= & (-1)^{m} \mathrm{j}^{n-l}(2 n+1) \\
& \times \sum_{p} \mathrm{j}^{p} g(m, l,-m, n, p) h_{p}\left(k r_{j i}\right) P_{p}\left(\cos \theta_{j i}\right),
\end{aligned}
$$

with $p=l+n, l+n-2, \ldots,|l-n|$ and the Gaunt coefficients given by

$$
\begin{aligned}
g(m, l,-m, n, p)= & (2 p+1) \sqrt{\frac{(l+m) !(n-m) !}{(l-m) !(n+m) !}} \\
& \times\left(\begin{array}{ccc}
l & n & p \\
0 & 0 & 0
\end{array}\right)\left(\begin{array}{ccc}
l & n & p \\
m & -m & 0
\end{array}\right) .
\end{aligned}
$$

Note that (.) in Eq. (16) does not refer to a matrix but a Wigner $3-j$ symbol. $^{23}$

Substituting Eqs. (14)-(16) into Eq. (11), applying the boundary condition $-\left.\left[\partial p_{\mathrm{tot}}\left(\mathbf{r}_{1}\right) / \partial r_{1}\right]\right|_{r_{1}=b}=\mathrm{j} \rho_{0} \omega U^{D}$ and $-\left.\left[\partial p_{\text {tot }}\left(\mathbf{r}_{2}\right) / \partial r_{2}\right]\right|_{r_{2}=a}=0$ at the surface of Sphere 1 and Sphere 2 in their own coordinate system, truncating the summations to a constant order $L$ and equating the coefficients of $P_{l}^{m}(\cos \theta) \cos \left[m\left(\varphi-\varphi_{D}\right)\right]$ yield the following coupled linear equations:

$$
\begin{aligned}
& C_{l m} h_{l}^{\prime}(k b) P_{l}^{m}\left(\cos \theta_{D}\right)+\sum_{n=m}^{L} D_{n m} P_{n}^{m}\left(\cos \theta_{D}\right) A_{m l}^{m n}\left(\mathbf{r}_{21}\right) j_{l}^{\prime}(k b) \\
& =-\mathrm{j} \rho_{0} c_{0} N_{l m} P_{l}^{m}\left(\cos \theta_{D}\right) \sum_{n=m}^{L} C_{n m} P_{n}^{m}\left(\cos \theta_{D}\right) \\
& \quad \times A_{m l}^{m n}\left(\mathbf{r}_{12}\right) j_{l}^{\prime}(k a)+D_{l m} h_{l}^{\prime}(k a) P_{l}^{m}\left(\cos \theta_{D}\right)=0,
\end{aligned}
$$

where $\operatorname{lm}=\{(0,0)(1,0)(1,1) \cdots(L, L)\}$ and $N_{l m}$ $=\left(2-\delta_{0 m}\right) U_{l 0} \sqrt{2 l+1 / 4 \pi}[(l-m) ! /(l+m) !]$. Equation (17) can be expressed in a more compact form by using matrix notations as

$$
\left[\begin{array}{cc}
\mathbf{S}_{1} & \mathbf{Q}_{21} \\
\mathbf{Q}_{12} & \mathbf{S}_{2}
\end{array}\right]\left[\begin{array}{l}
\mathbf{C} \\
\mathbf{D}
\end{array}\right]=\left[\begin{array}{l}
\mathbf{A}_{1} \\
\mathbf{A}_{2}
\end{array}\right]
$$

where $\mathbf{S}_{1}$ and $\mathbf{S}_{2}$ are $(L+1-m) \times(L+1-m)$ diagonal complex matrices, $\mathbf{Q}_{12}$ and $\mathbf{Q}_{21}$ are $(L+1-m) \times(L+1-m)$ complex matrixes, $\mathbf{C}, \mathbf{D}, \mathbf{A}_{1}$, and $\mathbf{A}_{2}$ are complex vectors of dimension $(L+1-m)$ for each $m$, and $m$ ranges from 0 to $L$. The elements of these matrixes and vectors are

$$
\begin{aligned}
& \mathbf{S}_{1}^{l}=h_{l}^{\prime}(k b) P_{l}^{m}\left(\cos \theta_{D}\right), \quad \mathbf{S}_{2}^{l}=h_{l}^{\prime}(k a) P_{l}^{m}\left(\cos \theta_{D}\right), \\
& \mathbf{Q}_{21}^{l n}=P_{n}^{m}\left(\cos \theta_{D}\right) A_{m l}^{m n}\left(\mathbf{r}_{21}\right) j^{\prime}{ }_{l}(k b), \\
& \mathbf{Q}_{12}^{l n}=P_{n}^{m}\left(\cos \theta_{D}\right) A_{m l}^{m n}\left(\mathbf{r}_{12}\right) j^{\prime}{ }_{l}(k a), \\
& \mathbf{A}_{1}^{l}=-\mathrm{j} \rho_{0} c_{0} N_{l m} P_{l}^{m}\left(\cos \theta_{D}\right), \quad \mathbf{A}_{2}^{l}=0 .
\end{aligned}
$$

Equation (18) has to be solved $L+1$ times to determine all the $C_{l m}$ and $D_{l m}$ coefficients. After that, the coefficients $C^{\prime}{ }_{l m}$ and $D^{\prime}{ }_{l m}$ in Eq. (12) can be obtained, and the sound pressure at each microphone can be calculated by Eq. (11). Then the performance of different beamforming algorithms with the speaker source model under the influence of the scattering can be simulated.

Apart from the commonly used array patterns, the DF is also utilized in this paper to numerically assess the directivity performance of the microphone array beamformers, which is defined as ${ }^{9}$

$$
\mathrm{DF}(k)=10 \log _{10}\left(\frac{\left|H\left(k, \theta_{l}, \varphi_{l}\right)\right|^{2}}{\frac{1}{4 \pi} \int_{0}^{2 \pi} \int_{0}^{\pi}|H(k, \theta, \varphi)|^{2} \sin \theta d \theta d \varphi}\right),
$$

where $\left(\theta_{l}, \varphi_{l}\right)$ is the look direction of the beamformer and $H(k, \theta, \varphi)$ is the array response.

\section{SIMULATIONS}

In this section, the effects of a near-field scatterer on the performance of linear microphone array beamformers are presented with numerical simulations on two typical beamformers, i.e., the DS and the superdirective beamformers. The DS beamformer has the benefit of the maximum robustness, ${ }^{1}$ while the superdirective beamformer has the benefit of the maximum directivity. ${ }^{9,14}$ Geometrical arrangements of the sound source, the rigid sphere and the microphone array are shown in Fig. 1. A diagonal loading factor of $\varepsilon=0.001$ is used for the robust superdirective beamformer design. ${ }^{14}$ In the following simulations, the microphone array is placed in the $x$ axis with the center being the origin of the coordinate system.

\section{A. Beamformers with the far-field plane wave scattering model}

Figure 2 depicts the time response of the plane wave scattering from a rigid sphere and shows what happens when a short plane wave pulse impinges on a rigid sphere, using similar calculation method as described in Ref. 20. The plane wave incident from the direction of $z>0$. Nine frames of the time response are listed sequentially from top left to bottom right. In each frame, the total sound field is calculated over an area of $x \in[-0.30 .3] \mathrm{m}$ and $z \in[-0.10 .5] \mathrm{m}$. The center of the rigid sphere is located at $\left[\begin{array}{lll}0 & 0 & 0.3\end{array}\right] \mathrm{m}$. In the time domain simulation, the radius of the rigid sphere $a$ is $0.1 \mathrm{~m}$, the sampling frequency is $12.8 \mathrm{kHz}$, and the time increment between each frame is three sampling intervals. It can be seen that the wavefront is delayed due to the influence of the scattering and the delay is mainly manifested in the area of $x$ $\in\left[\begin{array}{ll}-0.15 & 0.15\end{array}\right] \mathrm{m}$ and $z \in\left[\begin{array}{ll}-0.1 & 0.2\end{array}\right] \mathrm{m}$, i.e., the rear side of the rigid sphere. Therefore, it is reasonable to anticipate that the scatterer at the look direction of the array has the most influence on the beamforming performance. In the following simulations, the rigid sphere is placed in the broadside direction, i.e., $\theta_{s}=90^{\circ}$, with a distance of $0.3 \mathrm{~m}$ between the center of the rigid sphere and the center of the array. 

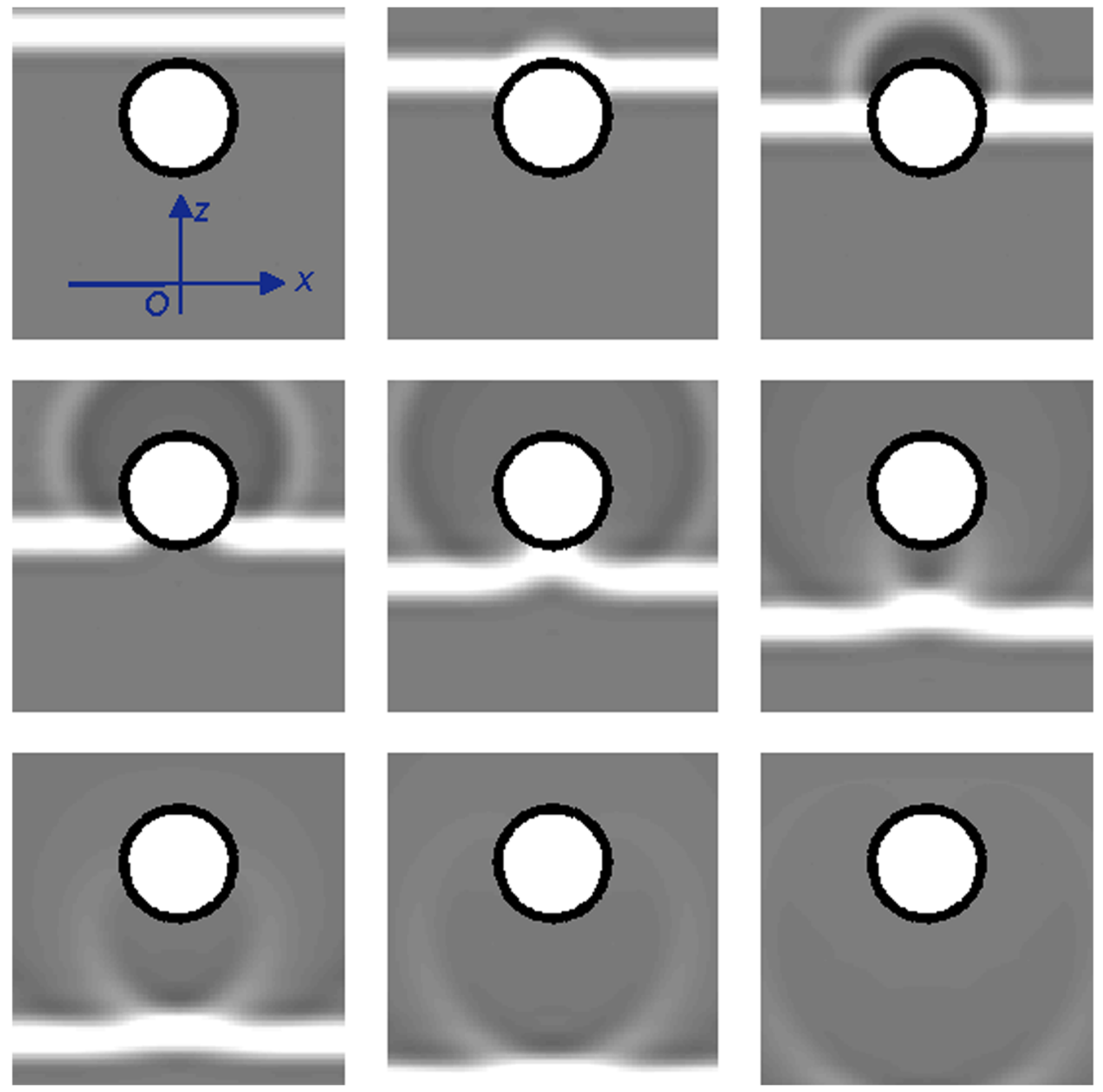

FIG. 2. (Color online) Time response of the plane wave scattering from a rigid sphere.
Figure 3 depicts the normalized array patterns with and without consideration of the rigid sphere scattering at 1.0 and $3.5 \mathrm{kHz}$. In this simulation, a five-element linear microphone array with element spacing $d=4 \mathrm{~cm}$ is used, and the radius of the sphere is $10 \mathrm{~cm}$. The results indicate that the beamwidth of both the DS and the superdirective beamformers becomes narrower when the rigid sphere scattering is considered. Because of the diffraction effect, the transmission time between the signal source and the microphone located at the rear side of the rigid sphere is increased as depicted in Fig. 2. This increases the phase differences between microphones, and leads to the increase of the effective element spacing and the effective aperture of the array. Therefore the beamwidth of the beamformer becomes narrower. Note that similar phenomenon has been noticed for microphone array mounted on a rigid sphere. ${ }^{18,19}$

Figure 4 depicts the normalized array patterns with the beamformers steered to the direction of $\theta=60^{\circ}$. It can be seen that the scatterer has little influence on the performance of the beamformer when the array is steered away from the rigid sphere. Therefore, in the following simulations, the beamformer is designed with broadside look direction.

Figure 5 depicts the DF of the far-field DS and superdirective beamformers with and without consideration of the rigid sphere scattering for different microphone number $M$, element spacing $d$ and rigid sphere radius $a$. It can be seen that when the size of the microphone array is no larger than that of the rigid sphere, the DF of both the DS and the superdirective beamformers becomes higher, as depicted in Figs. $5(\mathrm{a})-5(\mathrm{~b})$. When a plane wave is scattered by a rigid sphere, the sound energy is concentrated in the direction of the incident wave. ${ }^{22}$ This increases the sound pressure received by the microphones at the broadside direction for small-sized arrays, leading to a higher DF. When the size of the microphone array is close to that of the rigid sphere, the increased equivalent array aperture will lead to a narrower beamwidth and a higher DF, similar to the feature of the microphone array mounted on a rigid sphere. ${ }^{18,19}$ When the size of the array is significantly larger than that of the rigid sphere, rigid sphere scattering only influences a small part of the microphone array and there is hardly any variation of the DF, as depicted in Fig. 5(d).

An interesting phenomenon that should be noted in Fig. 5(c) is that the DF deteriorates under the influence of scattering. This is mainly caused by the increased side-lobe level. Although putting the sphere in the look direction of the beamformers causes the beamwidth to become narrower, it also cause some degradation to the side-lobe levels, similar to the circular array mounted on the rigid sphere. ${ }^{18}$ When the signal incidents from the main-lobe direction, the microphone array is located at the rear side of the rigid sphere, i.e., the shadow region as depicted in Fig. 6(a), and the beamwidth of the beamformer becomes narrower because of the increased equivalent array aperture. When signal incidents 


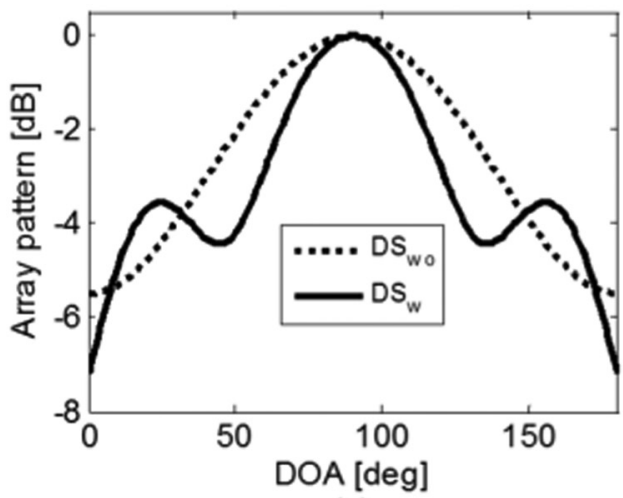

(a)

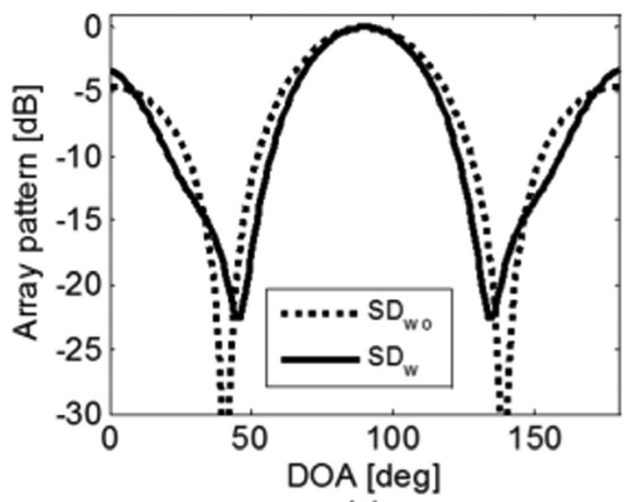

(c)

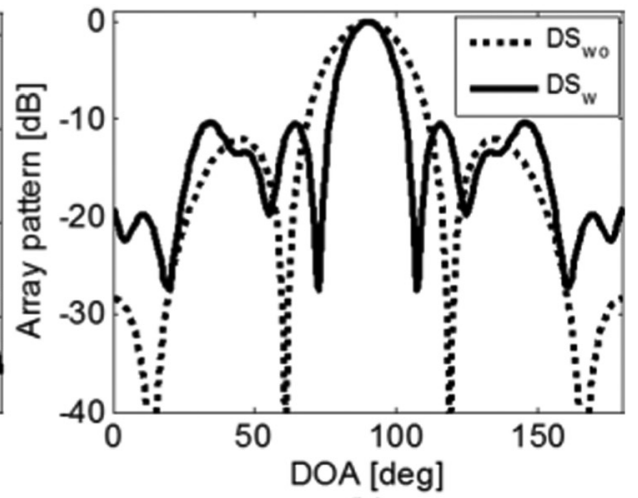

(b)

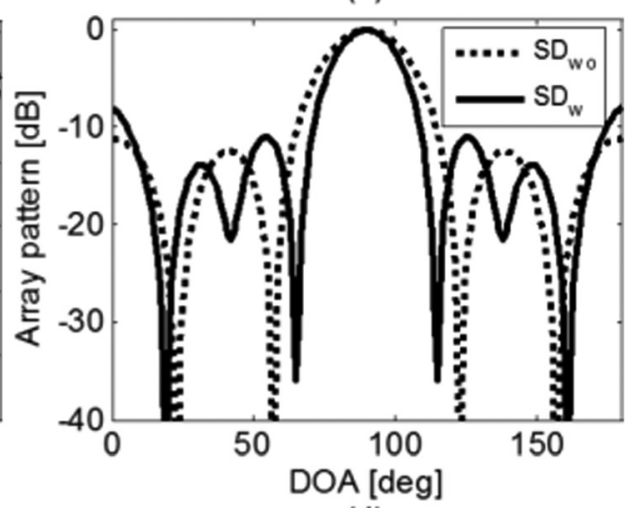

(d)
FIG. 3. When the beamformer is steered to the direction of $\theta=90^{\circ}$, array patterns for the far-field beamformer with (w) and without (wo) sphere scattering with $M=5, d=4 \mathrm{~cm}$, $a=10 \mathrm{~cm}$. (a) DS beamformer at $1.0 \mathrm{kHz}$, (b) DS beamformer at $3.5 \mathrm{kHz}$, (c) superdirective (SD) beamformer at $1.0 \mathrm{kHz}$, (d) superdirective beamformer at $3.5 \mathrm{kHz}$. from the side-lobe direction, most of the microphone elements will no longer be in the shadow region if the size of the microphone array is no larger than that of the rigid sphere, as depicted in Fig. 6(b). Therefore the increment of the side-lobe level is limited. However, when the size of the array is larger than that of the rigid sphere, the microphone array will be influenced by the shadow region when the signal incidents from the side-lobe direction, as depicted in

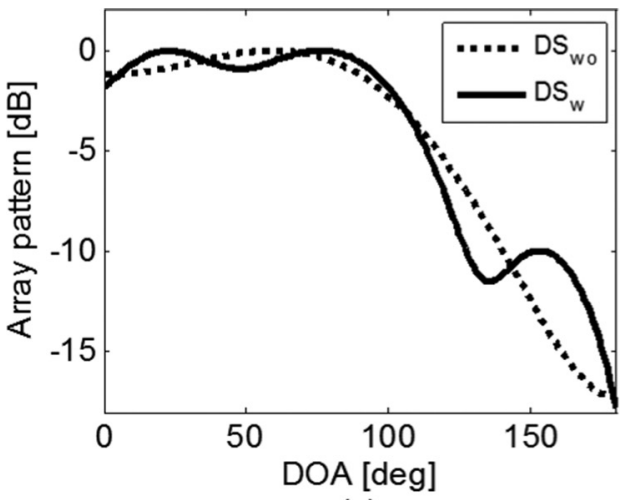

(a)

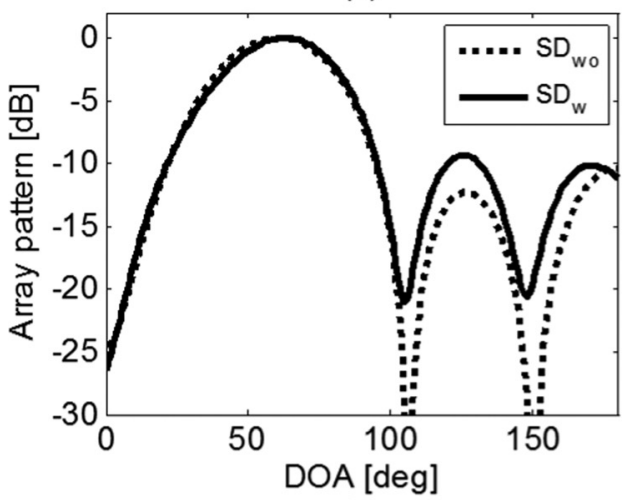

(c)

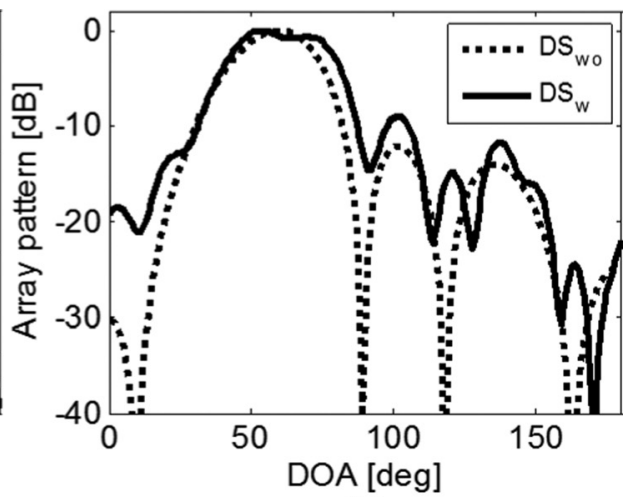

(b)

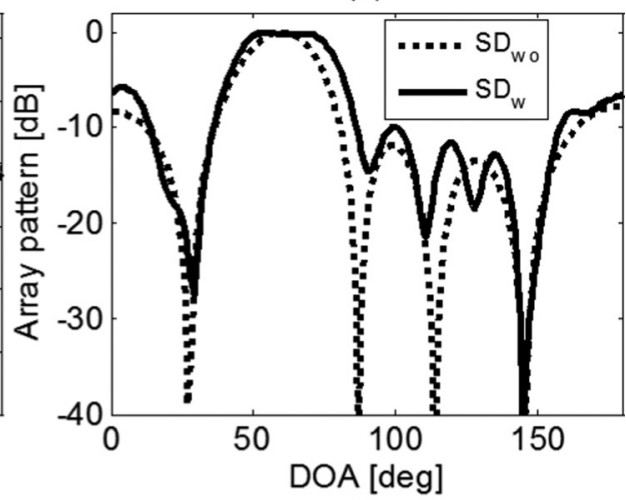

(d)
FIG. 4. When the beamformer is steered to the direction of $\theta=60^{\circ}$, array patterns for the far-field beamformer with (w) and without (wo) sphere scattering with $M=5, d=4 \mathrm{~cm}$, $a=10 \mathrm{~cm}$. (a) DS beamformer at $1.0 \mathrm{kHz}$, (b) DS beamformer at $3.5 \mathrm{kHz}$, (c) superdirective (SD) beamformer at $1.0 \mathrm{kHz}$, (d) superdirective beamformer at $3.5 \mathrm{kHz}$. 


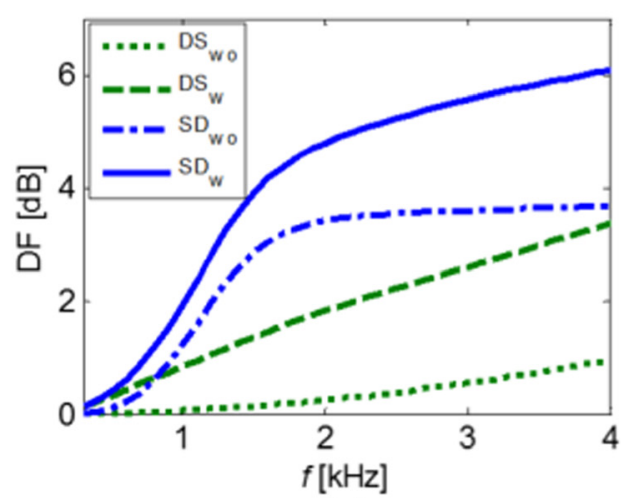

(a)

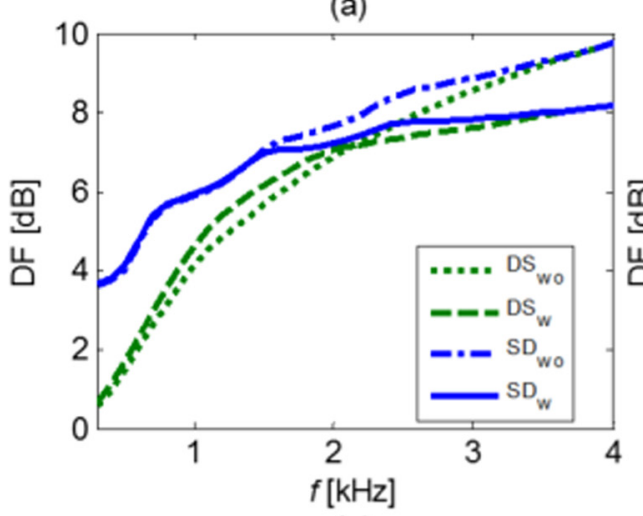

(c)

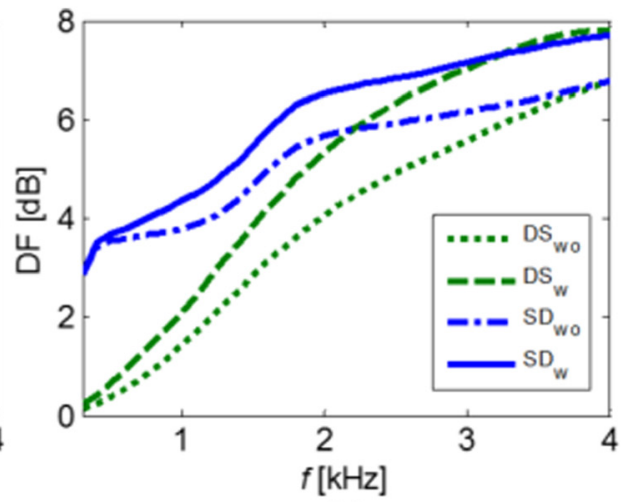

(b)

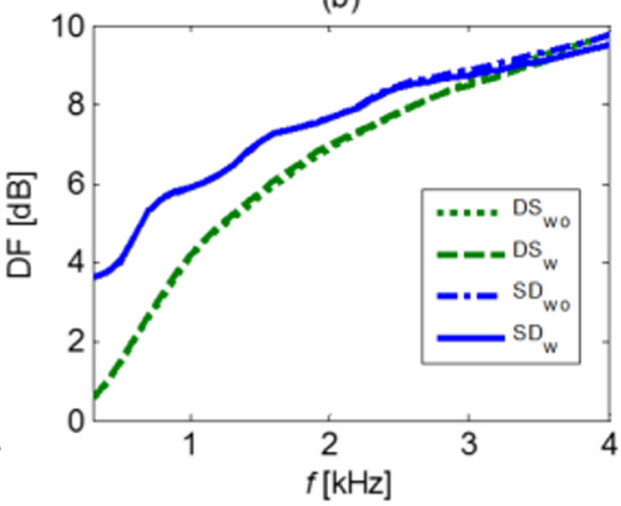

(d)
FIG. 5. (Color online) Directivity factor of the far-field DS and superdirective (SD) beamformers with (w) and without (wo) consideration of the sphere scattering. (a) $M=5, d=0.8 \mathrm{~cm}, a=10 \mathrm{~cm}$, (b) $M=5, \quad d=4 \mathrm{~cm}, \quad a=10 \mathrm{~cm}, \quad$ (c) $M=10, \quad d=4 \mathrm{~cm}, \quad a=10 \mathrm{~cm}, \quad(\mathrm{~d})$ $M=10, d=4 \mathrm{~cm}, a=5 \mathrm{~cm}$.
Fig. 6(b). The corresponding side-lobes would arise much higher at high frequencies and the DF of the corresponding beamformers becomes lower as depicted in Fig. 5(c).

\section{B. Near-field beamformers with the point source scattering model}

In the near-field beamformer simulation, the distance between the sound source and the center of the array is kept at $0.6 \mathrm{~m}$. Figure 7 depicts the DF of the near-field DS and superdirective beamformers with and without consideration of the rigid sphere scattering. For the near-field situation, the sound energy of the point source is also concentrated in the direction of the incident wave after rigid sphere scattering. ${ }^{22}$ Meanwhile, because of the diffraction around the rigid sphere, the transmission time between the point source and the microphone at the rear side of the rigid sphere is also

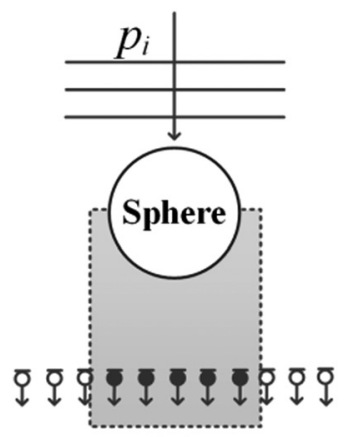

(a)

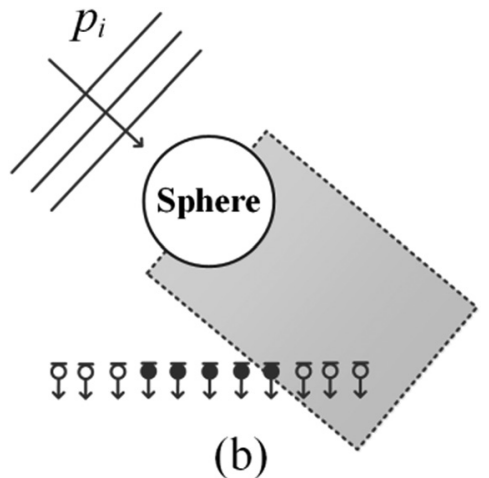

(b)
FIG. 6. (a) Signal incidents from the main-lobe direction. (b) Signal incidents from the side-lobe direction. increased. ${ }^{20}$ Therefore, the DF of both the DS and the superdirective beamformers becomes higher, when the size of the microphone array is no larger than that of the rigid sphere, as depicted in Figs. 7(a)-7(b).

Similar to the far-field situations, the beamformers suffer from the increased side-lobe level, when the size of the array is larger than that of the rigid sphere. The DF of the corresponding beamformers becomes lower at high frequencies as depicted in Fig. 7(c), where it can also be seen that the DF tends to decrease at high frequencies under the influence of scattering. Under the point source assumption, the rigid sphere scattering influences the sound field more evidently than that under the plane wave assumption. ${ }^{20}$ The corresponding side-lobe levels under the point source scenario tend to be higher at high frequencies. As a result, the corresponding DF decreases at high frequencies as depicted in Fig. 7(c). When the size of the array is significantly larger than that of the rigid sphere, there is hardly any variation of the DF, as depicted in Fig. 7(d).

\section{Near-field beamformers with the speaker scattering model}

In this simulations, the sound source is assumed to be a speaker model, the radius of the speaker is $b=0.1 \mathrm{~m}, \mu_{0}$ is $1.0 \mathrm{~m} / \mathrm{s}$ and $\theta_{0}$ is $13.2^{\circ}$. The distance between the mouth and the center of the array is kept at $0.6 \mathrm{~m}$. Figure 8 depicts the DF of the near-field DS and superdirective beamformer with and without consideration of rigid sphere scattering. Similar to the near-field situation with the point source, the DF of both the near-field beamformers becomes higher when the size of the microphone array is no larger than that of the 


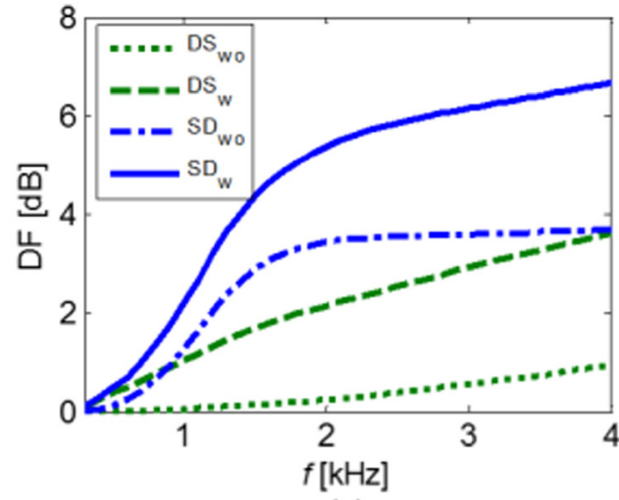

(a)

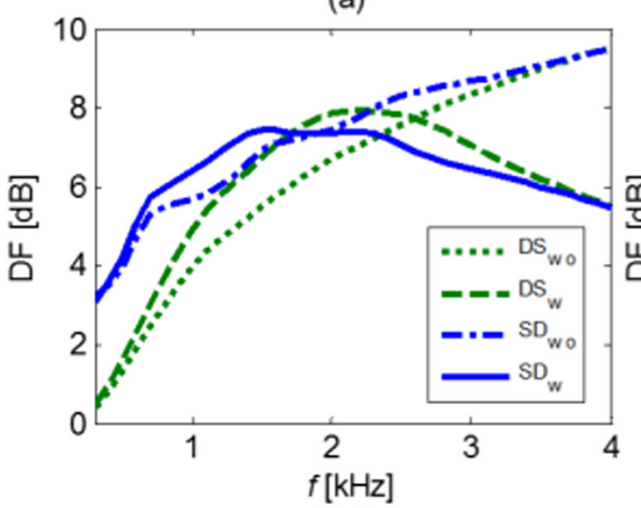

(c)

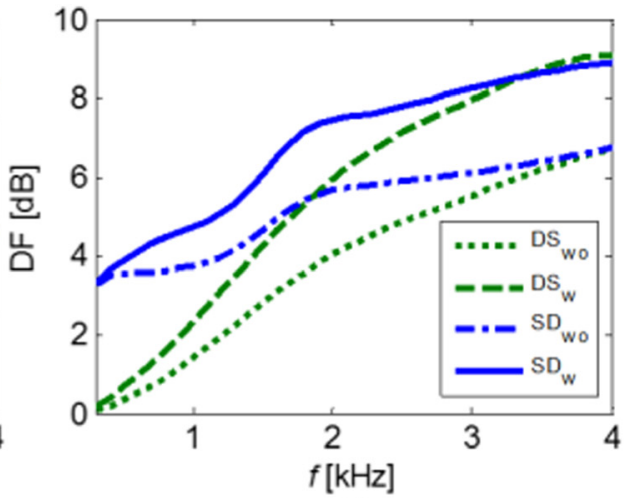

(b)

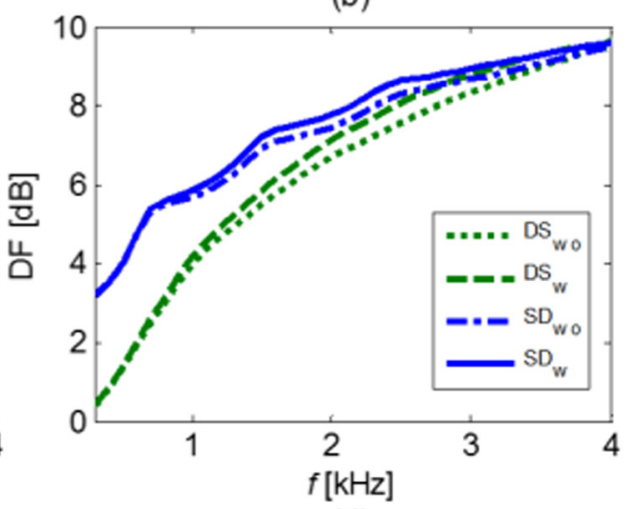

(d)
FIG. 7. (Color online) Directivity factor of the near-field DS and superdirective (SD) beamformers with $(\mathrm{w})$ and without (wo) consideration of the sphere scattering. (a) $M=5, d=0.8 \mathrm{~cm}, a=10 \mathrm{~cm}$, (b) $M=5, \quad d=4 \mathrm{~cm}, \quad a=10 \mathrm{~cm}, \quad$ (c) $M=10, \quad d=4 \mathrm{~cm}, \quad a=10 \mathrm{~cm}, \quad(\mathrm{~d})$ $M=10, d=4 \mathrm{~cm}, a=5 \mathrm{~cm}$. rigid sphere. When the size of the microphone array is larger than that of the rigid sphere, the corresponding DF tends to decrease at high frequencies and becomes lower than that without rigid sphere scattering. When the size of the microphone array is significantly larger than the rigid sphere, there is hardly any variance of the DF. Furthermore, when compared with Fig. 7, it can be found that the DF is nearly the same as those with a point source model. This indicates that the physical model of the sound source has little influence on the performance of the beamformer.

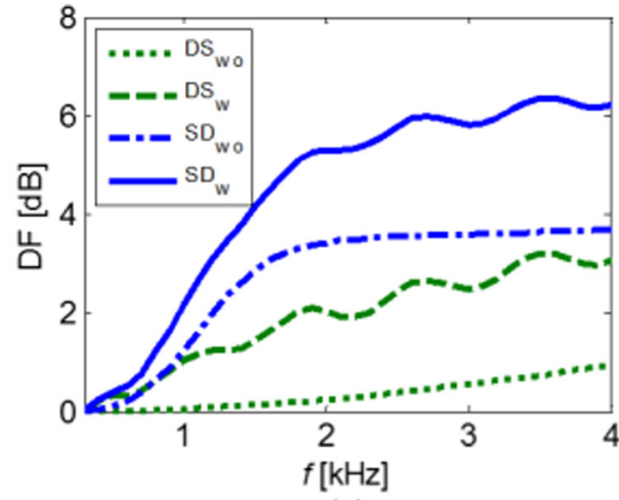

(a)

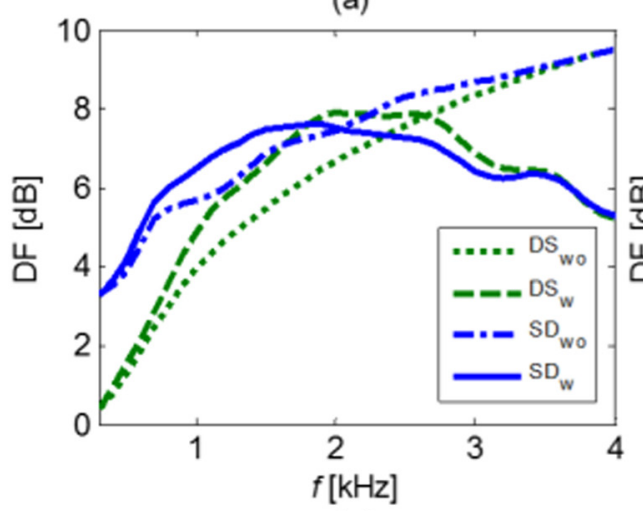

(c)

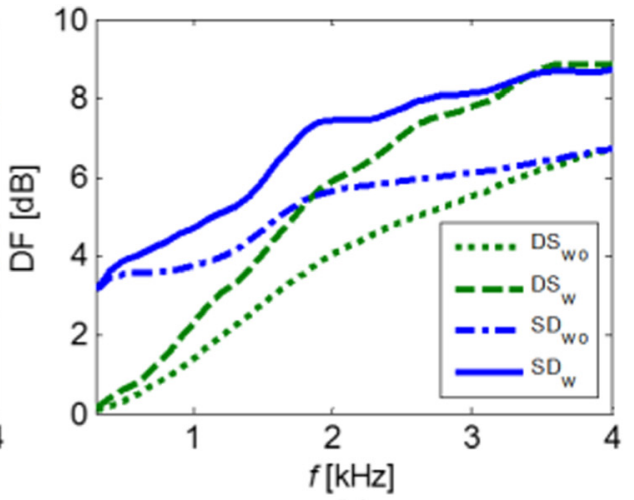

(b)

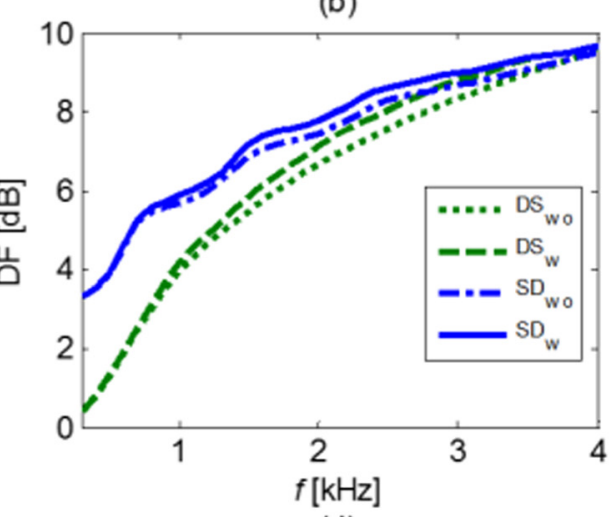

(d)
FIG. 8. (Color online) Directivity factor of the near-field DS and superdirective (SD) beamformers using the speaker model with (w) and without (wo) consideration of the sphere scattering. (a) $M=5, \quad d=0.8 \mathrm{~cm}, \quad a=10 \mathrm{~cm}, \quad$ (b) $M=5, d=4 \mathrm{~cm}, a=10 \mathrm{~cm}$, (c) $M=10$, $d=4 \mathrm{~cm}, \quad a=10 \mathrm{~cm}, \quad$ (d) $\quad M=10$, $d=4 \mathrm{~cm}, a=5 \mathrm{~cm}$. 


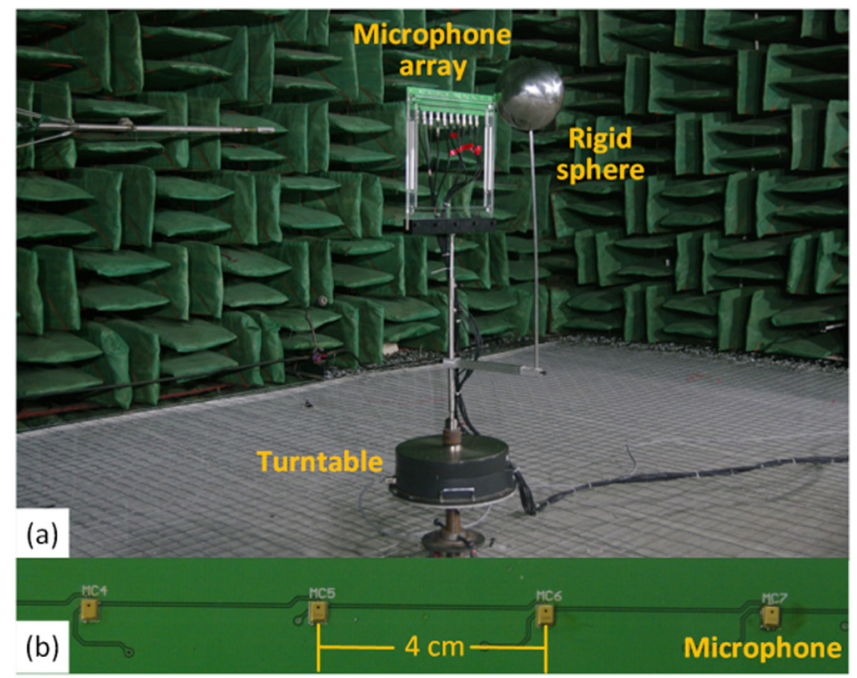

FIG. 9. (Color online) Experiment setup. (a) The panorama view of the experimental system, the rigid sphere is removable. (b) The microphones are spaced $4 \mathrm{~cm}$ apart.

From the above simulations, it can be concluded that the DF of the beamformers becomes higher, when the size of the microphone array is no larger than that of the rigid sphere. However, the drastic increment of the side-lobe level caused by the scattering leads to a lower DF of the beamformers when the size of the microphone array becomes larger. When the size of the array is significantly larger than that of the rigid sphere, the scattering only affects a small part of the microphone array and there is hardly any variation of the DF. It should be noted that although not shown in this paper, the same conclusions can be obtained when the beamformers are steered to other directions as long as the rigid sphere is placed in the look direction.

\section{EXPERIMENTS}

The experiments were carried out in an anechoic chamber as shown in Fig. 9. Two uniform linear microphone arrays consisting of 10 MEMS microphones with element spacing 4 and $0.8 \mathrm{~cm}$ were used. Two removable rigid spheres with radii of 10 and $5 \mathrm{~cm}$, respectively, were placed in the broadside direction of the array and rotated with the array synchronously. The distance between the center of the sphere and the microphone array is $0.3 \mathrm{~m}$. By fixing the position of the source and rotating the linear array around the center axis of the turntable, signals from different directions were recorded using an NI PXIe-4497 multi-channel measurement system with $16 \mathrm{kHz}$ sampling frequency and 24 bit sampling precision. As shown in Sec. III that similar beamformer variation is expected under different source model, only far-field source was used in the experiments.

Figure 10 depicts the normalized array pattern for the far-field DS and superdirective beamformers with $M=5$, $d=0.8 \mathrm{~cm}$, and $a=10 \mathrm{~cm}$ at 1.0 and $3.5 \mathrm{kHz}$. It can be seen that the beamwidth becomes significantly narrower when the rigid sphere is placed in the look direction of the beamformers. This coincides with the theoretical analysis since the array size is smaller than the size of the sphere. The directivity of the DS beamformer without consideration of the rigid sphere scattering at $3.5 \mathrm{kHz}$ seems a little abnormal. This is mainly caused by the directivity of the MEMS microphone

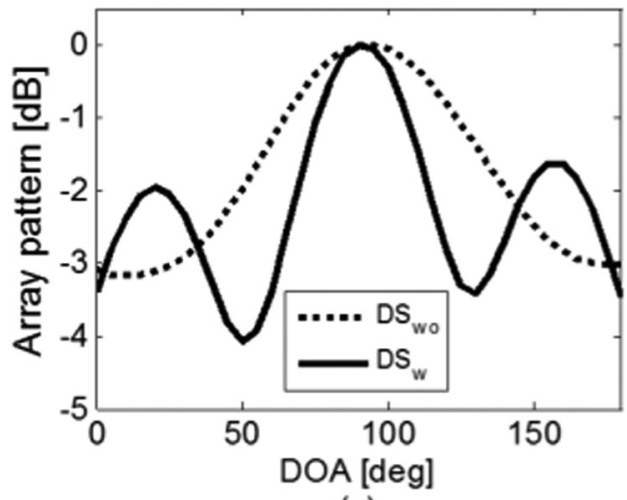

(a)

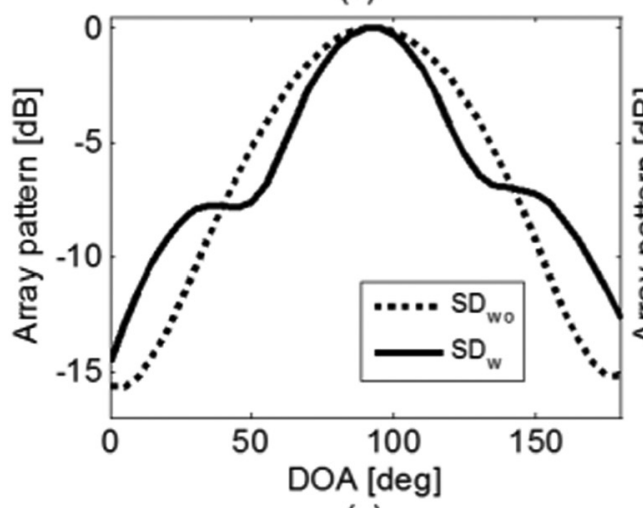

(c)

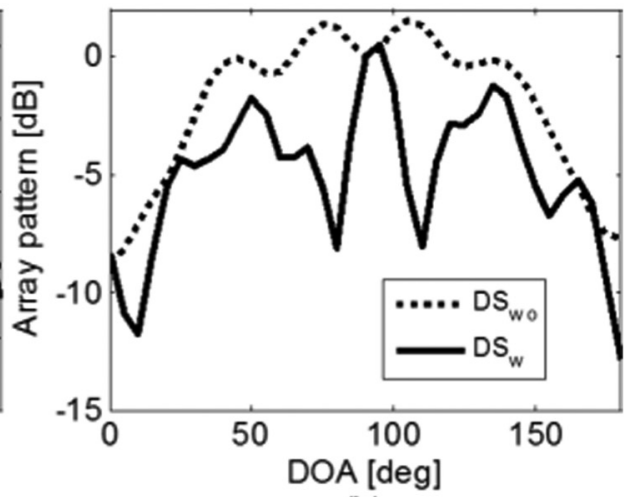

(b)

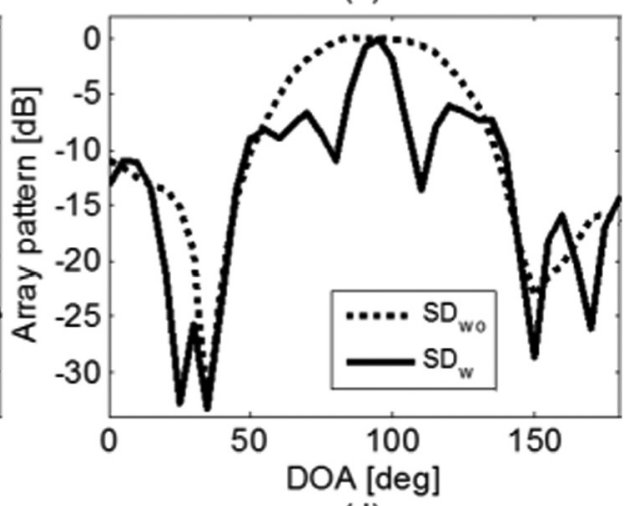

(d)
FIG. 10. Experiment results for the far-field beamformer with (w) and without (wo) sphere scattering with $M=5, d=0.8 \mathrm{~cm}, a=10 \mathrm{~cm}$. (a) DS beamformer at $1.0 \mathrm{kHz}$, (b) DS beamformer at $3.5 \mathrm{kHz}$, (c) superdirective (SD) beamformer at $1.0 \mathrm{kHz}$, (d) superdirective beamformer at $3.5 \mathrm{kHz}$. 


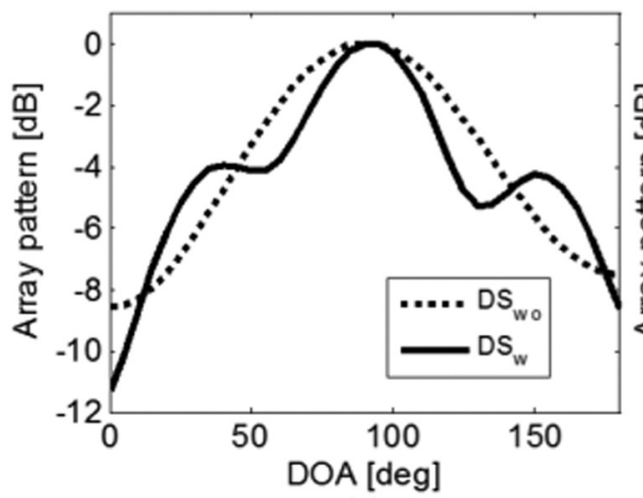

(a)

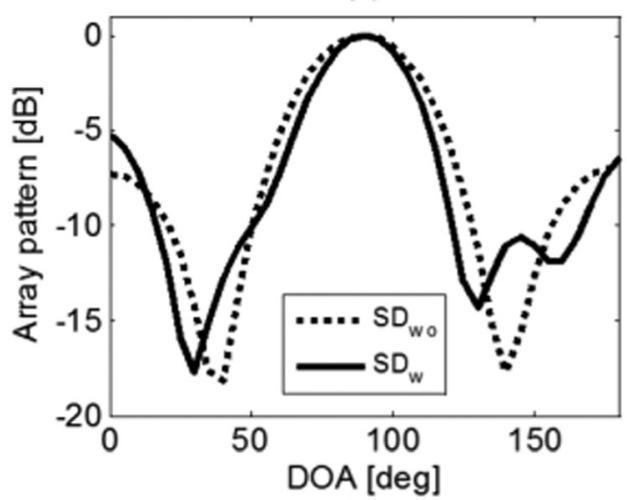

(c)

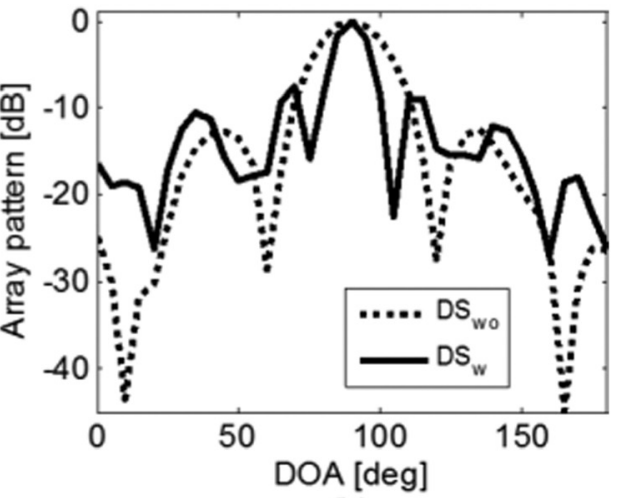

(b)

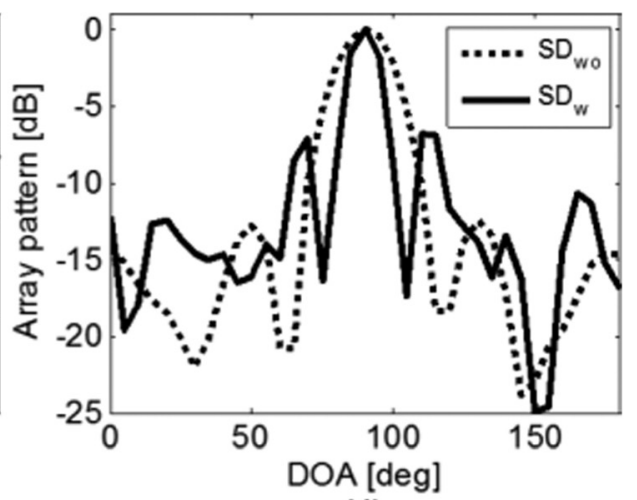

(d)
FIG. 11. Experiment results for the far-field beamformer with (w) and without (wo) sphere scattering with $M=5, d=4 \mathrm{~cm}, a=10 \mathrm{~cm}$. (a) DS beamformer at $1.0 \mathrm{kHz}$, (b) DS beamformer at $3.5 \mathrm{kHz}$, (c) superdirective (SD) beamformer at $1.0 \mathrm{kHz}$, (d) superdirective beamformer at $3.5 \mathrm{kHz}$. unit and the scattering of the panel in which the array is mounted.

Figure 11 depicts the normalized array pattern for the far-field DS and superdirective beamformers with $M=5$, $d=4 \mathrm{~cm}$, and $a=10 \mathrm{~cm}$ at 1.0 and $3.5 \mathrm{kHz}$. As expected from the simulation, the beamwidth is still narrower under the influence of the near-field scatterer when the array size is close to that of the sphere.

Figure 12 depicts the normalized array pattern for the far-field DS and superdirective beamformers with $M=10$,

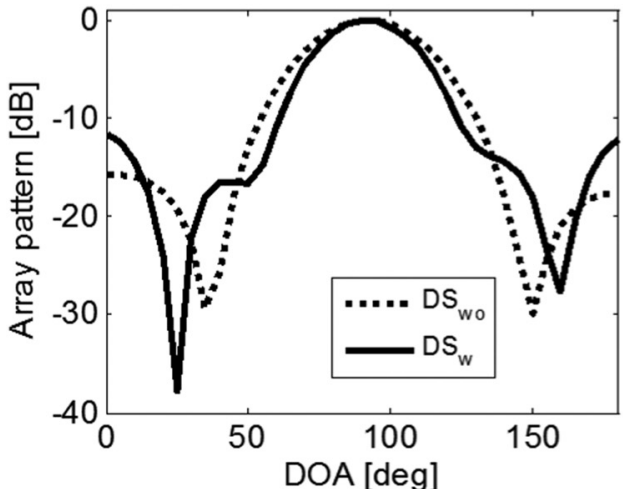

(a)

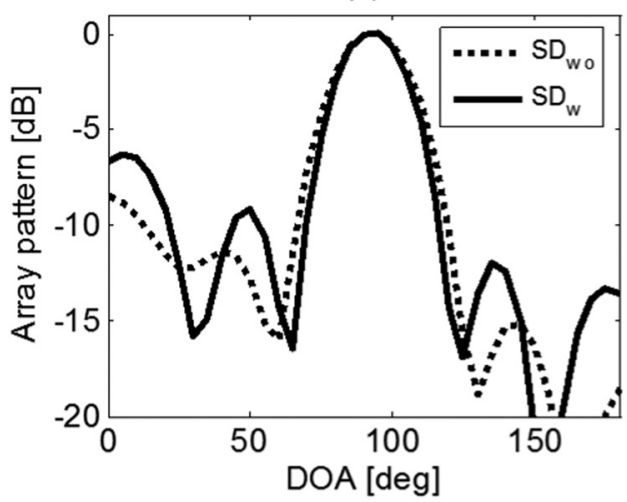

(c)

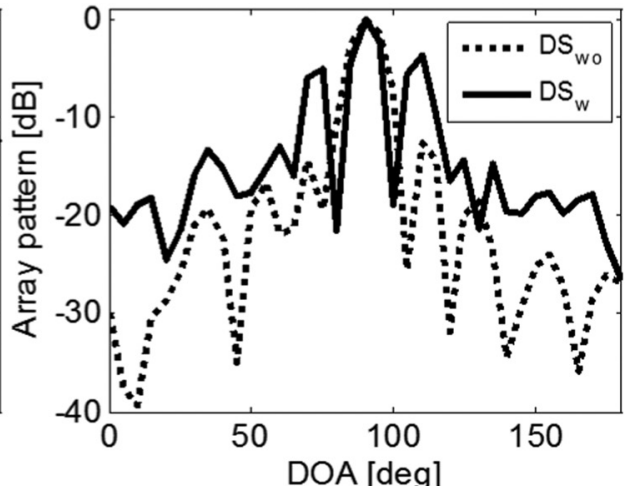

(b)

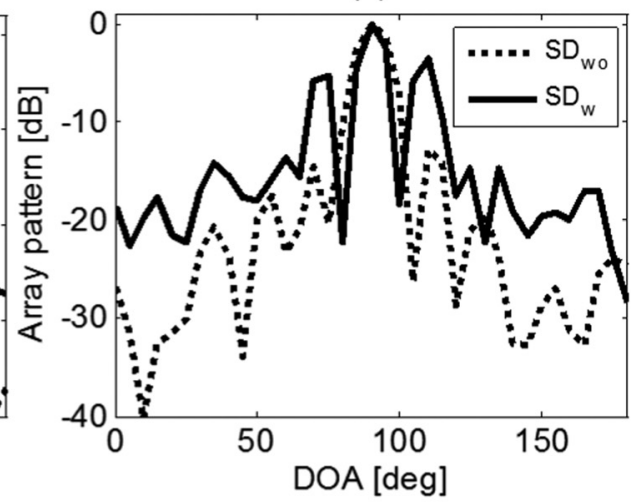

(d)
FIG. 12. Experiment results for the far-field beamformer with (w) and without (wo) sphere scattering with $M=10, d=4 \mathrm{~cm}, a=10 \mathrm{~cm}$. (a) DS beamformer at $1.0 \mathrm{kHz}$, (b) DS beamformer at $3.5 \mathrm{kHz}$, (c) superdirective (SD) beamformer at $1.0 \mathrm{kHz}$, (d) superdirective beamformer at $3.5 \mathrm{kHz}$. 


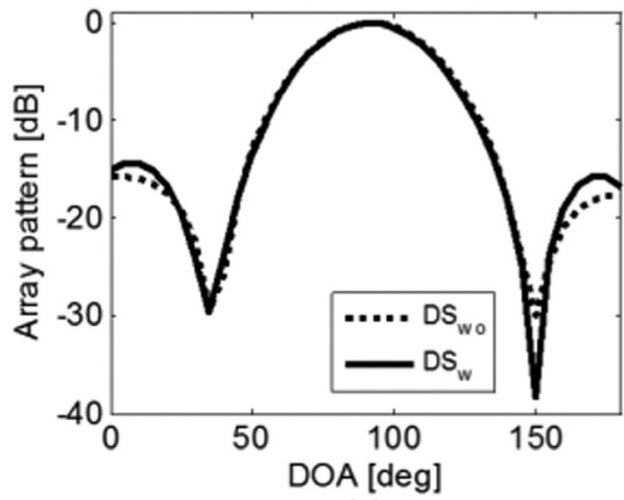

(a)

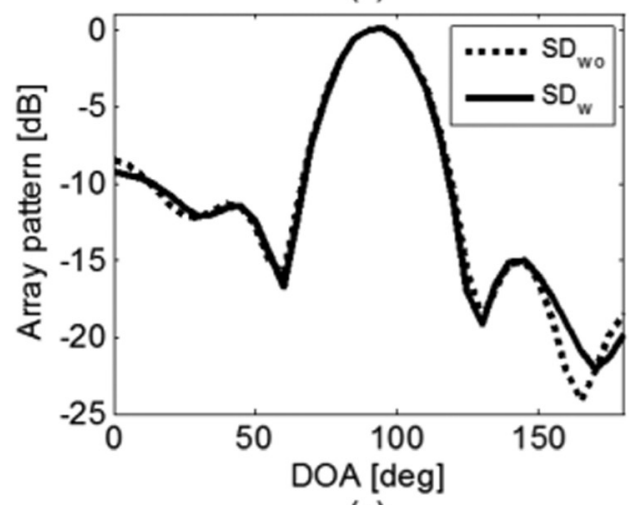

(c)

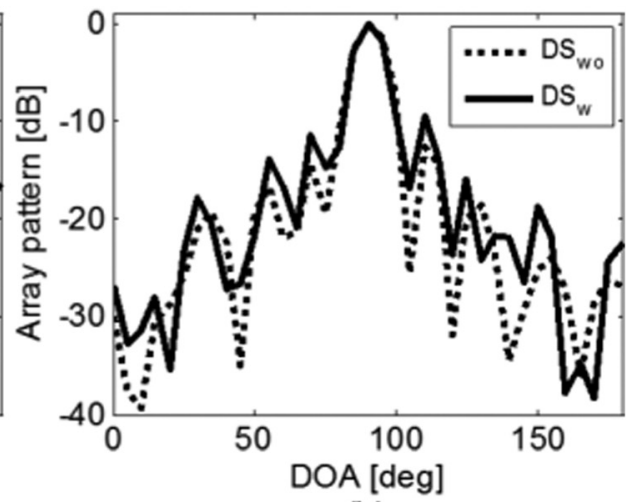

(b)

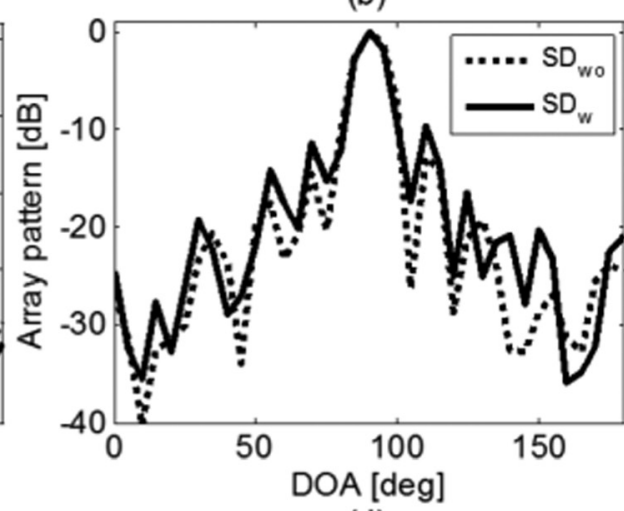

(d)
FIG. 13. Experiment results for the far-field beamformer with (w) and without (wo) sphere scattering with $M=10, d=4 \mathrm{~cm}, a=5 \mathrm{~cm}$. (a) DS beamformer at $1.0 \mathrm{kHz}$, (b) DS beamformer at $3.5 \mathrm{kHz}$, (c) superdirective (SD) beamformer at $1.0 \mathrm{kHz}$, (d) superdirective beamformer at $3.5 \mathrm{kHz}$. $d=4 \mathrm{~cm}$, and $a=10 \mathrm{~cm}$ at 1.0 and $3.5 \mathrm{kHz}$. When the array size is larger than that of the sphere, the beamwidth of the beamformers becomes narrower. However, the corresponding side-lobes of the beamformers become much higher, and this will lead to a lower DF.

Figure 13 depicts the normalized array pattern for the farfield DS and superdirective beamformers with $M=10$, $d=4 \mathrm{~cm}$, and $a=5 \mathrm{~cm}$ at 1.0 and $3.5 \mathrm{kHz}$. When the array size is significantly larger than that of the rigid sphere, there is hardly any influence on the array pattern of the beamformers. This meets the expectations from the theoretical analysis.

\section{CONCLUSIONS}

This paper investigates the influence of a near-field scatterer on the performance of a linear microphone array beamformer. The numerical models are established based on a rigid sphere scatterer and three different source models, i.e., the far-field plane wave, the near-field point source, and the near-field speaker model. From simulation results with two typical beamformers, the delay-and-sum and the superdirective beamformers, it can be found that the directivity factor of the beamformer becomes better when the array size is no larger than the size of the scatterer. This is a beneficial feature useful in designing small array with high directivity. When the array size is larger than that of the rigid sphere, the directivity factor drops down at high frequencies due to the rising side-lobes. However, when the array size is much larger than that of the rigid sphere, there is hardly any variation of the beamforming performance since the scattering only influence a small part of the array. Experiments in the anechoic chamber validate these remarks.

\section{ACKNOWLEDGMENTS}

This work was supported by National Science Foundation of China Grant No. 11374156.

${ }^{1}$ M. Brandstein and D. Ward, Microphone Arrays: Signal Processing Techniques and Applications (Springer Verlag, Berlin, 2001), pp. 1-38.

${ }^{2}$ K. Kumatani, J. McDonough, and B. Raj, "Microphone array processing for distant speech recognition: From close-talking microphones to far-field sensors," IEEE Signal Process. Mag. 29, 127-140 (2012).

${ }^{3}$ A. Avokh and H. R. Abutalebi, "Speech enhancement using linearly constrained adaptive constant directivity beam-formers," Appl. Acoust. 71, 262-268 (2010).

${ }^{4}$ S. Doclo and M. Moonen, "Design of far-field and near-field broadband beamformers using eigenfilters," Signal Process. 83, 2641-2673 (2003).

${ }^{5} \mathrm{~J}$. Meyer, "Beamforming for a circular microphone array mounted on spherically shaped objects," J. Acoust. Soc. Am. 109, 185-193 (2001).

${ }^{6}$ B. Rafaely, Y. Peled, M. Agmon, D. Khaykin, and E. Fisher, "Spherical microphone array beamforming," in Speech Processing in Modern Communication (Springer, Berlin, 2010), pp. 281-305.

${ }^{7}$ S. Yan, Y. Ma, and C. Hou, "Optimal array pattern synthesis for broadband arrays,” J. Acoust. Soc. Am. 122, 2686-2696 (2007).

${ }^{8}$ S. Doclo and M. Moonen, "Design of broadband beamformers robust against gain and phase errors in the microphone array characteristics," IEEE Trans. Signal Process. 51, 2511-2526 (2003).

${ }^{9} \mathrm{~S}$. Doclo and M. Moonen, "Superdirective beamforming robust against microphone mismatch," IEEE Trans. Audio, Speech, Lang. Process. 15, 617-631 (2007).

${ }^{10} \mathrm{M}$. Crocco and A. Trucco, "Design of robust superdirective arrays with a tunable tradeoff between directivity and frequency-invariance," IEEE Trans. Signal Process. 59, 2169-2181 (2011).

${ }^{11} \mathrm{H}$. Chen, W. Ser, and Z. L. Yu, "Optimal design of nearfield wideband beamformers robust against errors in microphone array characteristics," IEEE Trans. Circuits Syst. 54, 1950-1959 (2007).

${ }^{12}$ H. Chen, W. Ser, and J. Zhou, "Robust nearfield wideband beamformer design using worst case mean performance optimization with passband response variance constraint," IEEE Trans. Audio, Speech, Lang. Process. 20, 1565-1572 (2012). 
${ }^{13}$ R. C. Nongpiur, "Design of minimax broadband beamformers that are robust to microphone gain, phase, and position errors," IEEE/ACM Trans. Audio, Speech, Lang. Process. 22, 1013-1022 (2014).

${ }^{14}$ R. Berkun, I. Cohen, and J. Benesty, "Combined beamformers for robust broadband regularized superdirective beamforming," IEEE/ACM Trans. Audio, Speech, Lang. Process. 23, 877-886 (2015).

${ }^{15} \mathrm{~J}$. Meyer, "Microphone array for hearing aids taking into account the scattering of the head," in Proceedings of the IEEE Workshop on Applications of Signal Processing to Audio and Acoustics (2001), pp. 27-30.

${ }^{16} \mathrm{P}$. Gillett, M. Johnson, and J. Carneal, "A computational model for optimizing microphone placement on headset mounted arrays," in 123rd Convention of the Audio Engineering Society (2007), pp. 1-9.

${ }^{17}$ P. Gillett, M. Johnson, and J. Carneal, "Performance benefits of spherical diffracting arrays versus free field arrays," in Proceedings of the ICASSP (2008), pp. 5264-5267.

${ }^{18}$ G. A. Daigle, M. R. Stinson, and J. G. Ryan, "Beamforming with aircoupled surface waves around a sphere and circular cylinder," J. Acoust. Soc. Am. 117, 3373-3376 (2005).
${ }^{19}$ E. Tiana-Roig, F. Jacobsen, and E. Fernandez-Grande, "Beamforming with a circular array of microphones mounted on a rigid sphere," J. Acoust. Soc. Am. 130, 1095-1098 (2011).

${ }^{20}$ O. Kirkeby, P. A. Nelson, and H. Hamada, "Local sound field reproduction using two closely spaced loudspeakers,” J. Acoust. Soc. Am. 104, 1973-1981 (1998).

${ }^{21}$ Z. Lin, J. Lu, C. Shen, X. Qiu, and B. Xu, “Active control of radiation from a piston set in a rigid sphere," J. Acoust. Soc. Am. 115, 2954-2963 (2004).

${ }^{22}$ L. L. Beranek and T. Mellow, Acoustics: Sound Fields and Transducers (Elsevier, Amsterdam, 2012), pp. 487-533.

${ }^{23}$ X. Qiu, B. Masiero, and M. Vorländer, "Channel separation of crosstalk cancellation systems with mismatched and misaligned sound sources," J. Acoust. Soc. Am. 126, 1796-1806 (2009).

${ }^{24}$ K. M. Li, W. K. Lui, and G. H. Frommer, "The diffraction of sound by an impedance sphere in the vicinity of a ground surface," J. Acoust. Soc. Am. 115, 42-56 (2004).

${ }^{25} \mathrm{~B}$. Masiero and X. Qiu, "Two listeners crosstalk cancellation system modelled by four point sources and two rigid spheres," Acta Acust. Acust. 95, 379-385 (2009). 